\title{
Dilemas vividos pela equipe de enfermagem no cuidado ao paciente com COVID-19
}

\section{na UTI: Revisão integrativa}

\author{
Dilemmas experienced by the nursing team in patient care with COVID-19 in the ICU: Integrative \\ review
}

Dilemas vividos por el equipo de enfermería en la atención al paciente con COVID-19 en la UCI: revisión integradora

Anna Clara Carnaúba Marques ORCID: https://orcid.org/0000-0002-1612-8848 Universidade Federal de Alagoas, Brasil E-mail: claracarnaubaa@gmail.com

Eveline Lucena Vasconcelos ORCID: https://orcid.org/0000-0003-3522-6248 Universidade Federal de Alagoas, Brasil E-mail: evelinelucena@gmail.com Isabel Comassetto ORCID: https://orcid.org/0000-0002-2389-9384 Universidade Federal de Alagoas, Brasil E-mail: isabelcomassetto@ gmail.com

Raíssa Rafaella Santos Moreno da Silva ORCID: https://orcid.org/0000-0002-7265-9352 Universidade Federal de Alagoas, Brasil E-mail: raissarafaella13@ gmail.com

Thaís Honório Lins Bernardo

ORCID: https://orcid.org/0000-0002-8058-8400 Universidade Federal de Alagoas, Brasil E-mail: thais.bernardo@eenf.ufal.br

\begin{abstract}
Resumo
Objetivo: Identificar e analisar as evidências científicas disponíveis na literatura sobre os dilemas vividos pela equipe de enfermagem na assistência ao paciente com COVID-19 na UTI. Metodologia: Trata-se uma pesquisa de revisão integrativa, atendendo as recomendações PRISMA e desenvolvida em seis etapas: 1) identificação do tema e formulação da questão norteadora; 2) busca na literatura; 3) categorização dos estudos; 4) avaliação dos estudos incluídos; 5) interpretação dos resultados; 6) síntese do conhecimento. Resultados: A busca nas bases de dados: Scopus; Embase, ScienceDirect, Lilacs, Base de Dados em Enfermagem - BDENF, PubMed Central, MEDLINE, Web of Science e Cinahl. Publicados no período de janeiro de 2020 a abril de 2021. A busca alcançou 248.900 artigos, dos quais foram selecionados cinco artigos que atenderam aos critérios estabelecidos. Os dilemas identificados na literatura foram a escassez de mão de obra qualificada e especializada, carência de equipamentos de proteção individual, cargas de trabalho abusivas, estresse e medo, falha na comunicação com o paciente, exaustão física e emocional. Conclusão: Considera-se de extrema importância salientar essa realidade e buscar estratégias que favoreçam a diminuição dos dilemas vividos pelos profissionais de enfermagem que prestam assistência de enfermagem ao paciente com COVID-19 na UTI.
\end{abstract}

Palavras-chave: Cuidados de enfermagem; Pandemias; Infecções por coronavírus; Unidades de terapia intensiva.

\begin{abstract}
Objective: To identify and analyze the scientific evidence available in the literature on the dilemmas experienced by the nursing staff in patient care with COVID-19 in the ICU. Methodology: This is an integrative review research, meeting the PRISMA recommendations and developed in six stages: 1) identification of the theme and formulation of the guiding question; 2) literature search; 3) categorization of studies; 4) evaluation of included studies; 5) interpretation of results; 6) knowledge synthesis. Results: The search in the databases: Scopus; Embase, ScienceDirect, Lilacs, Nursing Database - BDENF, PubMed Central, MEDLINE, Web of Science and Cinahl. Published from January 2020 to April 2021. The search reached 248,900 articles, from which five articles that met the established criteria were selected. The dilemmas identified in the literature were the shortage of qualified and specialized labor, lack of personal protective equipment, abusive workloads, stress and fear, poor communication with the patient, physical and emotional exhaustion. Conclusion:
\end{abstract}


It is considered extremely important to highlight this reality and seek strategies that favor the reduction of dilemmas experienced by nursing professionals who provide nursing care to patients with COVID-19 in the ICU.

Keywords: Nursing care; Pandemics; Coronavirus infections; Intensive care units.

\section{Resumen}

Objetivo: Identificar y analizar la evidencia científica disponible en la literatura sobre los dilemas que vive el personal de enfermería en la atención al paciente con COVID-19 en la UCI. Metodología: Se trata de una investigación de revisión integradora, que cumple con las recomendaciones de PRISMA y se desarrolla en seis etapas: 1) identificación del tema y formulación de la pregunta orientadora; 2) búsqueda de literatura; 3 ) categorización de estudios; 4) evaluación de los estudios incluidos; 5) interpretación de resultados; 6) síntesis de conocimientos. Resultados: La búsqueda en las bases de datos: Scopus; Embase, ScienceDirect, Lilacs, Nursing Database - BDENF, PubMed Central, MEDLINE, Web of Science y Cinahl. Publicado desde enero de 2020 hasta abril de 2021. La búsqueda alcanzó 248,900 artículos, de los cuales se seleccionaron cinco artículos que cumplieron con los criterios establecidos. Los dilemas identificados en la literatura fueron escasez de mano de obra calificada y especializada, falta de equipo de protección personal, cargas de trabajo abusivas, estrés y miedo, mala comunicación con el paciente, agotamiento físico y emocional. Conclusión: Se considera de suma importancia resaltar esta realidad y buscar estrategias que favorezcan la reducción de los dilemas vividos por los profesionales de enfermería que brindan cuidados de enfermería a los pacientes con COVID-19 en la UCI.

Palabras clave: Atención de enfermería; Pandemias; Infecciones por coronavirus; Unidades de cuidados intensivos.

\section{Introdução}

Em dezembro de 2019, aconteceram os primeiros casos de Coronavirus Disease 2019 (COVID- 19) em Wuhan, China, sendo percebidos pela incidência de casos de um quadro de acometimento respiratório que frequentemente apresentava evolução para Pneumonia e/ou Severe Acute Respiratory Syndrome (SARS). Após investigações, foi encontrado um novo vírus nas secreções respiratórias de pacientes acometidos: o SARS-CoV-2, agente etiológico da COVID- 19. A partir da descoberta do "novo coronavírus", mais de 27 países confirmaram casos da doença e, em 11 de março de 2020, a Organização Mundial de Saúde (OMS) declarou uma pandemia, como resultado da rápida propagação do vírus e alto poder de transmissibilidade, por meio de gotículas e aerossóis (Lima et al., 2021).

Após a declaração da Pandemia de COVID-19, cada país implementou medidas de prevenção e controle, como uso obrigatório de máscara e isolamento social, e cada um enfrentou diversas situações com diferentes taxas de infecção e mortalidade. A rápida propagação da doença surpreendeu muitos sistemas de saúde que se desdobraram para fornecer leitos de Unidades de Terapia Intensiva (UTI), ventiladores mecânicos e Equipamentos de Proteção Individual (EPI) para profissionais de saúde e pacientes (Lee \& Lee; Artnez et al., 2020).

Em geral, os pacientes sintomáticos de COVID-19 apresentam: tosse, febre, fadiga, dispneia e mialgia, contudo, cerca de 20\% deles manifestam a forma grave podendo apresentar: Insuficiência Respiratória Aguda Hipoxêmica (IRpA), SARS, Síndrome do Desconforto Respiratório Agudo (SDRA) e Insuficiência Renal Aguda (IRA) tais quadros clínicos levam à necessidade de admissão em UTI. Há de se considerar que idosos e portadores de doenças de base têm maior risco de ter disfunção de múltiplos órgãos e de vir a óbito (Ramalho et al., 2020).

Para dar suporte aos pacientes acometidos pelo SARs-CoV-2 com quadro grave, estes são tratados na UTI, que tem como propósito promover a recuperação das funções vitais dos pacientes em um ambiente físico e psicológico adequado, conta com uma tecnologia avançada que possui equipamentos para a monitorização constante dos pacientes críticos e uma equipe especializada capaz de prestar cuidados aos diversos quadros clínicos e seus níveis de gravidade, 24 horas por dia (Ouchi et al., 2018).

Com o grande número de casos durante a pandemia, essa situação ocasionou enormes implicações, principalmente na assistência dos profissionais da saúde, com destaque para a Enfermagem, que desempenha papel singular na linha de frente no combate à COVID-19. Representado o maior grupo de profissionais da área da saúde atuando nesse contexto, enfrentando novos 
desafios diariamente (Fernandez at al.; De Sousa et al., 2020). Deste modo, é importante ter atenção e realizar estudos que promovam a assistência de qualidade, avaliando os profissionais e os capacitando, uma vez que neste setor, situações imprevistas e estressantes estão presentes, adversidades essas que podem prejudicar o atendimento, diminuindo sua qualidade, além de colocar em risco a vida do paciente (Rocha \& Lemos, 2017).

Conforme Borges et al. (2021), os enfermeiros são profissionais essenciais por dar assistência aos pacientes atuando na linha de frente da COVID-19 e em contextos já caracterizados por excesso de carga e ritmo de trabalho e pelo déficit de recursos humanos e materiais. O desconhecimento de uma nova doença, a preocupação de contraí-la e transmitir à família surgiram nesse período. Sendo uma situação nova, a pandemia acarretou a estes enfermeiros desafios associados ao trabalho e à busca de conhecimento. Araújo e Comassetto (2021), salientam que na gestão do serviço de saúde, o profissional necessita de domínio de todos os processos envolvidos - da logística à administração dos cuidados intra e extra unidade assistencial.

A enfermagem emergiu como prática social associada aos elementos que compõem a vida humana nos seus múltiplos aspectos, com base na prevenção, promoção e reabilitação da saúde. Os profissionais da enfermagem compreendem a maior categoria profissional da área da saúde, e ao permanecerem 24 horas ao lado dos pacientes, estão mais susceptíveis aos possíveis impactos psicológicos da pandemia (Ramos-Toescher et al., 2020).

Marins et al. (2020), traz que a equipe de enfermagem é indispensável na dinâmica da assistência para um bom prognóstico do paciente. Há uma pressão externa e interna para que profissionais de enfermagem desempenhem um cuidado de excelência, já que grande parte das intervenções ao paciente gravíssimo, são realizadas por eles.

Segundo Paixão et al. (2021), a pandemia trouxe um maior reconhecimento para a enfermagem quanto à sua atuação. Por outro lado, há uma desvalorização da categoria, decorrente das longas jornadas de trabalho sem equivalência salarial, inadequação de infraestrutura, más condições de trabalho e escassez de EPIs. Esses desafios existentes, somados a atual situação enfrentada pelos profissionais atuantes na linha de frente, geram o risco de desenvolver problemas psicológicos. Todos esses desafios interferem consequentemente na prestação do cuidado e na relação do enfermeiro com o paciente, tornando evidente a importância do trabalho em equipe e da educação permanente para que seja prestada uma assistência mais humanizada e de qualidade.

Dessa forma, enfatiza-se a relevância da atuação da equipe multidisciplinar como ponto de apoio para que esses profissionais possam seguir proporcionando um cuidado competente e de alta qualidade a seus clientes sem que isso interfira tão negativamente na sua vida (Cunha et al., 2020).

A crise global provocada pela COVID-19 demonstrou que, mesmo em países desenvolvidos, a estrutura hospitalar existente pode não ser capaz de atender a todos os que necessitem de tratamento médico. Em diversos momentos, países relataram que o número de pacientes contaminados que precisavam de unidades de terapia intensiva (UTI) e aparelhos de ventilação mecânica excedeu o número de equipamentos disponíveis e de profissionais especializados (Marmelstein \& Morozowski, 2020).

Todas as características supracitadas, próprias da realidade da UTI, foram maximizadas devido ao surgimento da pandemia e, a partir de então, foi avaliada a capacidade de internação em UTI e do isolamento físico de portadores da COVID19. Estimando-se 5\% de internação destes pacientes em UTI, fez-se necessária a ampliação das UTIs. Houveram mudanças nas rotinas assistenciais, pois foi reforçada a necessidade de cumprir as diretrizes de prevenção de infecção relacionada aos dispositivos invasivos, redução do tempo do banho no leito e alterações no rodízio de reposicionamento no leito. As medidas de precaução, paramentação e higienização das mãos precisaram ser reforçadas a partir de capacitações (Gerolin et al., 2020).

Nesse cenário, muitas pessoas que teriam que ser tratadas com a devida internação e eventual utilização de aparelhos de ventilação mecânica faleceram pela incapacidade do sistema de oferecer tratamento adequado. É uma dramática situação de 
escassez, em que a demanda de pacientes supera a oferta de leitos de UTI disponíveis, acarretando mortes que seriam evitáveis em um momento de normalidade (Marmelstein \& Morozowski, 2020).

Campos e Canabrava (2020) explicam que para cada leito necessário para o atendimento à pacientes com COVID-19, são também exigidos novos fluxos de organização de acesso, novos equipamentos, especialmente ventiladores mecânicos, uma rede elétrica e de gases capaz de suportar essa sobrecarga, insumos em quantidade e qualidade adequadas e, sobretudo, força de trabalho capacitada para atender ao crescente número de casos complexos e muito graves. Reforça ainda que, uma rede de serviços com graves distorções na alocação geográfica dos recursos assistenciais, inclusive de leitos de UTI, tem tensionado enormemente o sistema de saúde mundial.

Percebe-se que a realidade das UTIs quanto à sua quantidade e composição (material e humano) eram insuficientes de maneira bem significativa antes da pandemia e após, se tornou um problema maior o qual precisou ser resolvido às pressas para atender à demanda de paciente. Diante do exposto, esta pesquisa teve como objeto de estudo: "as adversidades na assistência de enfermagem ao paciente com COVID-19 na UTI", guiado pela pergunta norteadora do estudo: "Quais foram as adversidades na assistência de enfermagem ao paciente com COVID-19 na UTI?" Portanto, este estudo apresenta-se relevante para as investigações com o foco na melhoria da assistência de enfermagem se justifica por evidenciar as principais adversidades encontradas pela enfermagem que atua na assistência ao paciente crítico na UTI durante a atual pandemia de COVID-19, tendo em vista que o número de estudos publicados com este foco ainda está em construção, por ser uma situação pandêmica nova.

Ressalta-se que é essencial conhecer as adversidades a fim de criar soluções e estratégias que deem suporte técnico e psicológico que contribuirão para uma melhoria não só na assistência ao paciente, como também na qualidade da atuação profissional. Portanto, este estudo tem como objetivo identificar e analisar as evidências científicas disponíveis na literatura sobre os dilemas vividos pela equipe de enfermagem na assistência ao paciente com COVID-19 na UTI.

\section{Metodologia}

Trata-se de uma Revisão Integrativa (RI) de literatura científica, construída a partir de artigos publicados em bases de dados, capazes de responder à questão da pesquisa. Segundo Mendes et al. (2019), sintetizar o conhecimento é uma metodologia científica que resume evidências de vários estudos sobre uma questão específica, identificando lacunas em pesquisa e sugerindo novos estudos, a fim de embasar a tomada de decisão na saúde. Acrescentam que a RI é um método que permite essa síntese de conhecimento por meio de processo sistematizado e rigoroso.

De acordo com Sousa et al. (2017), a RI permite contribuir com a Enfermagem com o intuito de melhorar a prestação de cuidados e oferecer informações amplas sobre um assunto, estabelecendo assim, um extenso corpo de rigor metodológico e conhecimento, além de promover a diminuição de erros e vieses a partir de uma abordagem rigorosa.

Esta RI, atendeu as recomendações PRISMA e foi estruturada e construída em 6 etapas pré-estabelecidas: 1) escolha do tema e da questão norteadora da pesquisa, utilizando o método PICo ( P-problema/ I- Interesse; Co- Contexto); 2) definição dos critérios de inclusão e exclusão e busca dos estudos primários nas bases de dados; 3 ) extração de dados de cada estudo primário e organização dos incluídos na revisão; 4) avaliação crítica dos estudos primários; 5) síntese e discussão dos resultados da revisão e 6) apresentação da revisão integrativa (Mendes \& Silveira \& Galvão, 2019).

Os critérios de inclusão determinados foram: artigos em português, inglês e espanhol que foram publicados no período de janeiro de 2020 a abril de 2021, que estão disponíveis gratuitamente nas bases de dados escolhidas e que são capazes de trazer contribuições para responder à questão norteadora deste estudo. E os de exclusão foram: cartas ao editor, monografias, teses, entrevistas, editoriais, estudos com desenho metodológico fragilizado e artigos que não abordam o tema definido por esta revisão. 
As bases de dados escolhidas para realização das buscas deste estudo foram: Scopus; Embase, ScienceDirect, Literatura Latino-Americana e do Caribe em Ciências da Saúde - Lilacs, Base de Dados em Enfermagem - BDENF, PubMed Central, Medical Literature Analysis and Retrieval System Online - MEDLINE, Web of Science e Cumulative Index to Nursing and Allied Health Literature - Cinahl.

Os descritores utilizados foram: "Nursing care"; "Pandemics"; "Coronavirus infections" e "Intensive Care Units", agrupados pelo operador booleano "AND”. Tais descritores fazem parte da coletânea dos Descritores em Ciências da Saúde (DECS). As buscas foram realizadas nos meses de abril a junho de 2021, com todos os descritores combinados e feitas nos três idiomas da pesquisa: inglês, português e espanhol. Além disso, foram realizadas buscas adicionais com combinações de descritores dois a dois, com o foco de encontrar mais estudos para a amostra final desta RI. Por fim, cada estudo da amostra final foi classificado quanto ao nível de evidência científica, segundo o método de Nedel e Silveira (2016) que descreve sete níveis de evidência, explicados abaixo.

Os níveis de evidência científica, segundo o método de Nedel e Silveira (2016), foram descritos em 7 níveis diferentes: nível 1 - revisão sistemática/ metanálise, nível 2 - ensaio clínico randomizado (ECR), nível 3 - ensaio clínico não randomizado (quase experimental), nível 4 - estudo de coorte, nível 5 - caso-controle, nível 6 - estudos de prevalência (transversais) e nível 7 - série de casos; os quais definem o nível 1 com maior evidência científica e consequentemente, o 7 como o nível de menor evidência científica.

\section{Resultados}

Nas buscas realizadas nas bases de dados pré-determinadas, foi encontrado um quantitativo elevado de artigos, no entanto, a expressiva maioria não abordava a temática definida para esta revisão integrativa. De forma geral, as combinações com os descritores dois a dois resultaram em números altos de estudos achados, principalmente na língua inglesa, no entanto, estes artigos não se encaixaram nos critérios estabelecidos e, principalmente, não responderam à questão norteadora desta revisão, fugindo do tema central, sendo, portanto, excluídos.

Por ser, atualmente, um tema de grande relevância torna-se uma prioridade de pesquisa em esfera mundial, os estudos que abordam a temática da COVID- 19 crescem exponencialmente, refletindo o número alto de publicações encontradas a partir destas buscas. Entretanto, o primeiro resultado apontado por esta revisão é a disparidade entre o número de artigos encontrados e a quantidade que compõe a amostra final, representando os artigos que abordam as dificuldades da Enfermagem na assistência ao paciente acometido por COVID- 19 no âmbito da UTI, trazendo a atenção para a necessidade de maiores estudos com este foco de investigação. A trajetória de busca e seleção dos estudos da amostra final desta revisão foi explanada no Fluxograma 1. 
Figura 1 - Fluxo do processo de seleção dos estudos para revisão, 2021.

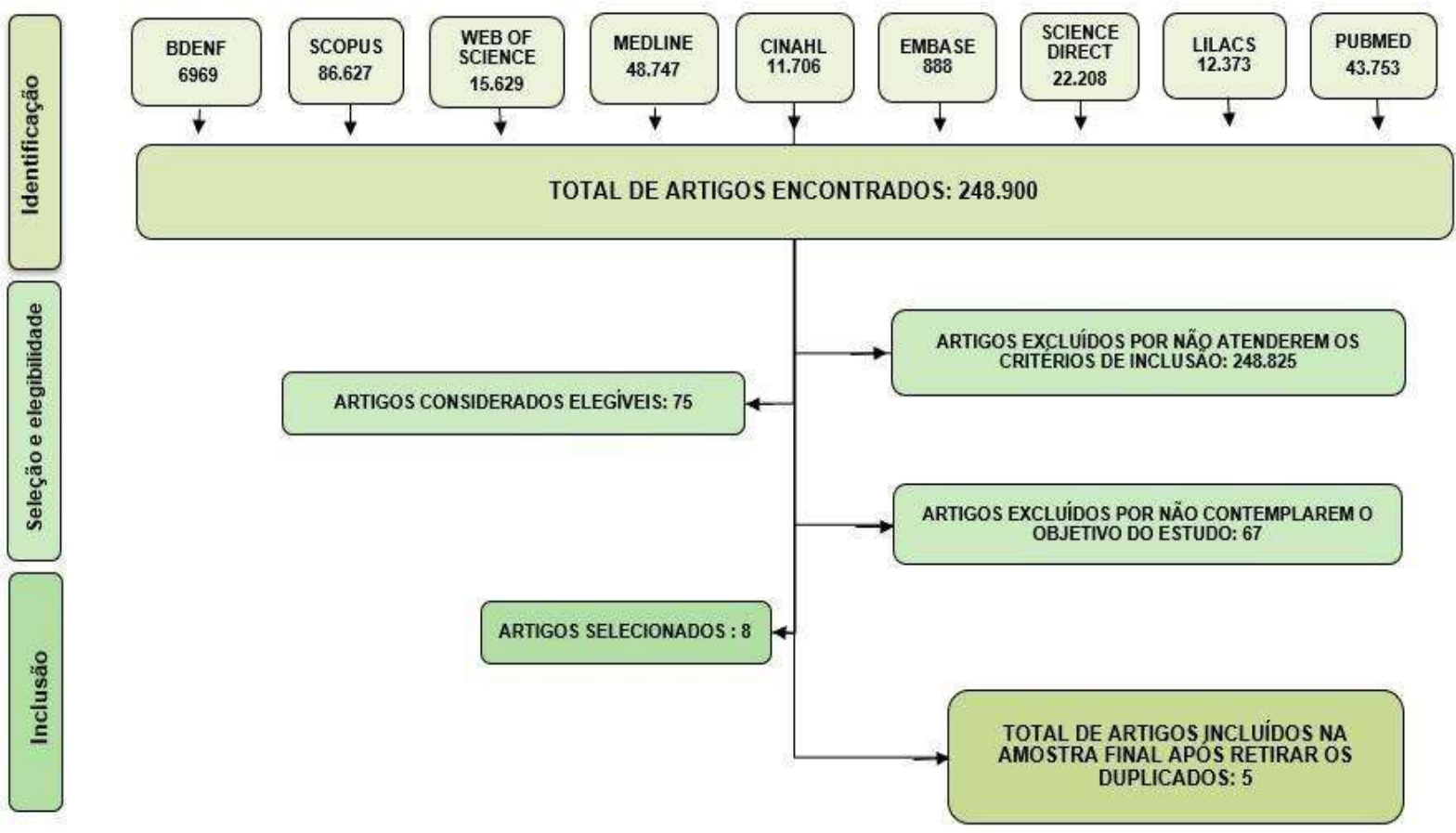

Fonte: Autores (2021).

É possível observar no Fluxograma 1, que as bases apresentaram uma quantidade de 248.900 estudos, porém o número de artigos selecionados para a amostra final desta revisão foi de apenas cinco, justificado pelo motivo supracitado em relação às combinações dos descritores e a escassez de estudos direcionados para este foco da temática. Nas bases de dados Embase, Science Direct e Medline não foi selecionado artigo algum para compor a amostra final, pois os achados não se adequaram aos critérios de inclusão e/ou trazem outras linhas de conteúdos em suas abordagens.

Por fim, das 9 bases selecionadas, foram escolhidos artigos de apenas 6 bases e, é necessário destacar que os artigos que foram encontrados em mais de uma base foram contabilizados apenas uma vez. Por conseguinte, a amostra final desta RI é composta por cinco artigos que estão apresentados no Quadro 1, com a identificação, título, objetivos, bases de dados que foram encontrados, o periódico de publicação, tipo de estudo, local de realização e o nível de evidência científica.

Quadro 1 - Detalhamento dos artigos selecionados na amostra final da revisão. Brasil, 2021.

\begin{tabular}{|c|c|c|c|c|}
\hline $\begin{array}{l}\text { Identificação/Título do } \\
\text { Artigo } \\
\begin{array}{l}\text { Ano e idioma de } \\
\text { publicação }\end{array}\end{array}$ & Objetivos & $\begin{array}{l}\text { Bases de dados e } \\
\text { Periódicos }\end{array}$ & $\begin{array}{l}\text { Tipo de estudo } \\
\text { Local de Pesquisa }\end{array}$ & $\begin{array}{l}\text { Nível de } \\
\text { Evidência }\end{array}$ \\
\hline $\begin{array}{l}\text { A01 } \\
\text { Otimização dos cuidados } \\
\text { intensivos na assistência ao } \\
\text { paciente com COVID-19 }\end{array}$ & $\begin{array}{l}\text { Refletir acerca das estratégias } \\
\text { para a otimização dos cuidados } \\
\text { intensivos na assistência ao } \\
\text { paciente com COVID-19. }\end{array}$ & $\begin{array}{l}\text { BDENF e Lilacs } \\
\text { Revista } \\
\text { Enfermagem em } \\
\text { foco (Brasília) }\end{array}$ & $\begin{array}{l}\text { Estudo teórico-reflexivo, que } \\
\text { resgata as considerações atuais } \\
\text { acerca da gestão do cuidado } \\
\text { intensivo no contexto da pandemia. }\end{array}$ & $\begin{array}{l}\text { VI } \\
\text { (Nedel \& } \\
\text { Silveira, } \\
\text { 2016) }\end{array}$ \\
\hline 2020 - Português & & & & \\
\hline
\end{tabular}




\begin{tabular}{|c|c|c|c|c|}
\hline $\begin{array}{l}\text { A02 } \\
\text { Critical care nursing during } \\
\text { the COVID-19 pandemic: a } \\
\text { story of resilience. } \\
2020 \text { - Inglês }\end{array}$ & $\begin{array}{l}\text { Evidenciar a importância de } \\
\text { recursos para apoiar } \\
\text { enfermeiras de UTI e outras } \\
\text { equipes de saúde para } \\
\text { gerenciar o estresse e } \\
\text { promover o bem-estar durante } \\
\text { a pandemia do COVID-19, } \\
\text { bem como, sinalizar a } \\
\text { necessidade de pesquisas sobre } \\
\text { a temática. }\end{array}$ & $\begin{array}{l}\text { Cinahl } \\
\text { British Journal of } \\
\text { Nursing }\end{array}$ & $\begin{array}{l}\text { Relato de experiência de uma } \\
\text { enfermeira de UTI que traz } \\
\text { algumas reflexões sobre o cuidado } \\
\text { aos pacientes com COVID-19 e } \\
\text { correlaciona sua experiência vivida } \\
\text { com o conceito de resiliência. }\end{array}$ & $\begin{array}{l}\text { VII } \\
\text { (Nedel } \\
\text { Silveira, } \\
\text { 2016) }\end{array}$ \\
\hline $\begin{array}{l}\text { A03 } \\
\text { Intensive care nurses' } \\
\text { experiences during the } \\
\text { COVID-19 } \\
\text { pandemic: A qualitative } \\
\text { study } \\
2020 \text { - Inglês }\end{array}$ & $\begin{array}{l}\text { Explorar e descrever as } \\
\text { experiências } \\
\text { e percepções de enfermeiras } \\
\text { que trabalhavam em uma UTI } \\
\text { durante a pandemia global de } \\
\text { COVID-19. }\end{array}$ & $\begin{array}{l}\text { Scopus, Web of } \\
\text { Science, PubMed } \\
\text { The Journal of } \\
\text { British } \\
\text { Association of } \\
\text { Critical Care } \\
\text { Nurses }\end{array}$ & $\begin{array}{l}\text { Pesquisa qualitativa, utilizando } \\
\text { uma abordagem empírica e técnicas } \\
\text { de análise indutiva de conteúdo, } \\
\text { realizada com enfermeiras de um } \\
\text { ensino superior em um hospital da } \\
\text { Espanha. }\end{array}$ & $\begin{array}{l}\text { V } \\
\text { (Nedel } \\
\text { Silveira, } \\
\text { 2016) }\end{array}$ \\
\hline $\begin{array}{l}\text { A04 } \\
\text { Challenges experienced by } \\
\text { ICU nurses throughout the } \\
\text { provision of care for } \\
\text { COVID-19 patients: A } \\
\text { qualitative study } \\
2021 \text { - Inglês }\end{array}$ & $\begin{array}{l}\text { Explorar os desafios } \\
\text { vivenciados pelos enfermeiros } \\
\text { de UTI ao longo da prestação } \\
\text { de cuidados aos pacientes com } \\
\text { COVID-19. }\end{array}$ & $\begin{array}{l}\text { Web of Science } \\
\text { Journal of Nursing } \\
\text { Management }\end{array}$ & $\begin{array}{l}\text { Estudo qualitativo descritivo, } \\
\text { foram selecionados } 17 \text { enfermeiros } \\
\text { que atuam em unidades de } \\
\text { terapia intensiva por amostragem } \\
\text { intencional no centro da cidade de } \\
\text { Urmia, Irã. Os dados foram } \\
\text { coletados por meio de entrevistas } \\
\text { semiestruturadas face a face. Após } \\
\text { a gravação e transcrição das } \\
\text { entrevistas, os conceitos foram } \\
\text { extraídos por meio do método de } \\
\text { análise de conteúdo. }\end{array}$ & $\begin{array}{l}\text { V } \\
\text { (Nedel } \\
\text { Silveira, } \\
\text { 2016) }\end{array}$ \\
\hline $\begin{array}{l}\text { A05 } \\
\text { Nurses' perceptions and } \\
\text { demands regarding COVID- } \\
19 \text { care delivery in } \\
\text { critical care units and } \\
\text { hospital emergency services } \\
2021 \text { - Inglês }\end{array}$ & $\begin{array}{l}\text { Identificar necessidades } \\
\text { relacionadas com segurança, } \\
\text { organização, tomada de } \\
\text { decisão, comunicação e } \\
\text { necessidades psico- } \\
\text { socioemocionais percebidas } \\
\text { por enfermeiros de cuidados } \\
\text { intensivos e emergências na } \\
\text { região de Madrid, Espanha, } \\
\text { durante a fase aguda da crise } \\
\text { epidémica. }\end{array}$ & $\begin{array}{l}\text { Web of Science, } \\
\text { PubMed } \\
\text { Intensive and } \\
\text { Critical Care } \\
\text { Nursing } \\
\text { (International } \\
\text { Journal } \\
\text { Research of } \\
\text { Practise) }\end{array}$ & $\begin{array}{l}\text { Estudo transversal (a primeira fase } \\
\text { de um estudo de métodos mistos) } \\
\text { com enfermeiros de cuidados } \\
\text { intensivos e emergências de } 26 \\
\text { hospitais públicos de Madrid, } \\
\text { utilizando um questionário online. }\end{array}$ & $\begin{array}{l}\text { VI } \\
\text { (Nedel } \\
\text { Silveira, } \\
\text { 2016) }\end{array}$ \\
\hline
\end{tabular}

Fonte: Autores (2021).

Estes artigos selecionados passaram por análise criteriosa a partir dos critérios de inclusão e exclusão definidos e foram lidos minuciosamente a fim de averiguar se os resultados apresentados são relevantes para este estudo e se o percurso metodológico descrito foi cumprido. 


\section{Falta de enfermeiros capacitados e especializados em terapia intensiva}

O A01 declarou que a pandemia potencializou a necessidade de capacitações. Isto ficou evidente, pois no momento em que mais precisou-se ficou perceptível a baixa quantidade de enfermeiros especializados em terapia intensiva. Já o A02 ressalta o pouco tempo para capacitação desde que a pandemia surgiu e que enfermeiras de UTI precisaram treinar enfermeiras de serviços de pequena e média complexidade para que estas pudessem assumir as responsabilidades de uma unidade de terapia intensiva.

No A03, é destacada a falta de enfermeiros com especialização em UTI e este foi um relato de enfermeiras de UTI de um ensino superior de um hospital na Espanha, que vivenciaram o início da pandemia do COVID-19.

O A04 aborda no tema "ineficiência da organização no apoio aos enfermeiros" a escassez de mão de obra qualificada, visto que isso interfere diretamente na carga de trabalho. No A05 os enfermeiros com especialização declararam ter menos medo de cometer erros e tinham um maior conhecimento. $28,2 \%$ dos enfermeiros que participaram da pesquisa relataram ainda não ter pessoal de enfermagem suficiente para a assistência.

\section{Desgaste físico, emocional e psicológico dos profissionais de enfermagem}

Já no A01 é enfatizada a necessidade de monitoramento do desgaste emocional e físico dos profissionais, pois a grande quantidade de pacientes graves de diversas faixas etárias; o dinamismo do cuidado; a alta taxa de letalidade; o uso ininterrupto de EPIS causando lesões e a limitação da comunicação com os pacientes são fatores que podem causar sofrimento psicológico nesses profissionais e que dificultam sua prestação da assistência. O A02 afirmou que os riscos psicológicos de uma pandemia não tem como ser evitados, mas que ser resiliente ajuda a superar os desafios e obstáculos nesse período de grande tensão.

O medo da morte de parentes e a predisposição à depressão e ao estresse agudo prolongado também foram ressaltados no A02. O medo foi a emoção mais relatada também no A03 por esses profissionais, somado à sensação de dar o seu melhor e não obter resultados esperados no tratamento do paciente, causando assim, um sentimento de frustração e insuficiência.

O A04 traz que o esgotamento físico dos trabalhadores da enfermagem foi citado em conjunto com os danos à pele causados pelos EPIs, bem como o peso que é trabalhar com eles. O descontrole hormonal que está diretamente ligado ao emocional, também foi evidenciado. Desconhecer a doença e seu prognóstico, a não existência de uma vacina até então, o medo de infectar familiares e amigos, a baixa vontade de comparecer aos plantões e até o arrependimento de ser enfermeiro foram emoções descritas pelos profissionais relacionadas ao tópico "vivendo com incertezas". Sobretudo, a carga psicológica se sobressaiu nos depoimentos, visto que acabou interferindo no desempenho pessoal dos indivíduos, afetando claramente suas vidas.

O A05 mostra que 37,5\% dos enfermeiros que participaram da pesquisa têm medo de se infectar, 62,8\% têm medo de infectar outras pessoas e 46,5\% sempre pensam na possibilidade de serem portadores assintomáticos. 44,9\% relataram ainda, exaustão emocional ao fim da jornada de trabalho, reforçando a difícil realidade vivida pela equipe de enfermagem em todo o mundo.

\section{Limitação de comunicação e contato com os pacientes}

O A01 afirma que a limitação da comunicação e a necessidade de distanciamento, interferem drasticamente na qualidade da assistência e na saúde mental dos profissionais. A identidade dos profissionais é velada pela necessidade do uso de grande quantidade de EPIs prejudicando a interação e comunicação entre profissionais e pacientes. Para minimizar esses efeitos, iniciativas para resgatar a humanização da assistência à saúde e superar o distanciamento estabelecido, têm sido utilizadas, como por exemplo, a criação de crachás "humanizados”, com fotos que destacam o rosto e o nome dos profissionais. 
Desta maneira, o A02 também mostra que foi preciso estabelecer novas maneiras de comunicação através da tecnologia para se comunicar tanto com funcionários, como com os pacientes e familiares. O A02 afirma ainda que as habilidades de comunicação da equipe de enfermagem estão constantemente sendo testadas e é um processo interativo transformador que perpassa pelas etapas de aprendizado, reflexão e reconfiguração.

O A03 reforça que a minimização do contato com o paciente, que se deu pela necessidade de isolamento, e a falta de proximidade prejudicam o desempenho ideal de cuidado, visto que limita o desenvolvimento das técnicas e o entendimento dos sentimentos do paciente.

\section{Extensão de perdas de vida e lidar com más notícias}

Lidar com más notícias e perdas de vida está presente no cotidiano dos enfermeiros da UTI, contudo, com a pandemia essa situação se intensificou a partir da alta taxa de letalidade do vírus e a grande quantidade de pacientes graves de diversas faixas etárias, como ficou evidenciado no A01 e no A02. É evidente que o enfermeiro intensivista precisa ter um domínio sobre a comunicação de más notícias visto que isso interfere diretamente em como os familiares e o paciente irão encarar o progresso da doença. Contudo, o A04 mostra que a falta de conhecimento sobre o prognóstico, a falta de especificidade dos medicamentos, a via de transmissão desconhecida, a apresentação clínica pouco clara, tornaram a situação vaga e incerta na qual trouxe insegurança e falta de controle dos profissionais, dificultando o gerenciamento emocional da extensão de perdas de vida.

O A02 reforça que os enfermeiros de UTI precisam ser resilientes diante da necessidade de saber separar as emoções pessoais das dos pacientes e familiares para fornecer-lhes o suporte necessário. Ressalta ainda que há uma preocupação maior com o risco e mortalidade da família e amigos do que a do próprio enfermeiro e com a pandemia essa realidade se agravou.

\section{Aumento da carga de trabalho}

O A02 ressalta que gerenciar grandes escalas foi um grande desafio para os enfermeiros e que houve aumento na carga de trabalho devido a necessidade de enfermeiras treinarem e supervisionarem enfermeiras de Nível 01 (Enfermaria) para cuidar do Nível 03 (cuidados intensivos) e Nível 02 (alta dependência). Segundo o A03, o aumento da carga de trabalho se deu pela falta de profissionais especializados em UTI e o aumento exponencial do número de pacientes.

Sem licença, escassez de mão de obra de enfermagem e pesados turnos de trabalho, indicaram a excessiva carga de trabalho dos enfermeiros que prestam cuidados aos pacientes com COVID-19 na UTI, de acordo com o A04.

O A05 mostra que 28,2\% relataram não ter pessoal de enfermagem suficiente para a assistência. A excessiva proporção paciente-enfermeiro também teve destaque nas percepções individuais dos enfermeiros que participaram deste estudo.

\section{Má gestão e escassez de EPIs}

O A01 mostra que há preocupação acerca da escassez e/ou inadequação dos EPI, somada à exposição de profissionais dos grupos de risco que não obtiveram respaldo para o afastamento das atividades de trabalho.

Enfermeiras que participaram da pesquisa no A03 afirmaram que má gestão de material de proteção individual tem sido um ponto negativo dessa situação pandêmica, causando um transtorno na assistência ao paciente com COVID-19 e que a aquisição insuficiente e inadequada de EPIs de acordo com os protocolos existentes, tem causado ansiedade.

Já as enfermeiras que contribuíram com a pesquisa do A04 destacaram que a quantidade EPIs por muitas vezes foi insuficiente e que havia discriminação na distribuição destes, os quais iam em maior quantidade em melhor qualidades para os profissionais médicos. 


\section{Discussão}

Na atual conjuntura mundial, o enfermeiro atua em várias vertentes, tornando-se protagonista na assistência em saúde e vem evidenciando a importância da classe no enfrentamento da doença, trazendo um reconhecimento das atividades desempenhadas pela equipe de enfermagem no desenvolvimento dos cuidados e promoção de saúde. Seu destaque se deu devido, principalmente, à proximidade com o paciente na assistência, por gerenciar setores e/ou unidades de saúde, realizar o planejamento e funcionalidade da estrutura física e construir protocolos e fluxos de cuidado (Paixão et al., 2021; Bitencourt et al., 2020).

Foram ressaltadas diversas situações vivenciadas no dia a dia da equipe de Enfermagem, em especial pelos Enfermeiros que atuam em UTI e lideram as tomadas de decisão frente aos cuidados no tratamento dos pacientes com quadro grave de COVID-19. Pôde-se perceber que as dificuldades apontadas pelos estudos da amostra final são semelhantes, contudo, cada local de pesquisa as descreve e lida de uma maneira singular, de acordo com suas vivências e necessidades.

Nessa realidade, o A01 enfatiza a necessidade de capacitações devido às dificuldades em detectar algumas situações, uma vez que para atuar na UTI são necessários conhecimentos específicos que permeiam lidar com a alta complexidade dos casos e por vezes não haviam profissionais treinados para tal e precisaram aprender na prática devido à necessidade de massa profissional.

Segundo Melo et al. (2019), estudos revelam que a falta de especialização na área de atuação pode ser uma das causas da omissão de cuidados, visto que o profissional não sabe como proceder naquele momento. Borges et al. (2017) diz que o dimensionamento do pessoal de enfermagem na UTI não corresponde ao efetivo real para a categoria de enfermeiras e a carência de profissionais especializados em terapia intensiva era alta, o que acarreta na sobrecarga de trabalho na unidade.

Outros destaques trazidos pelo A01 foram os desgastes físico e emocional da equipe de enfermagem, tendo como principais motivos: a grande quantidade de pacientes graves de diversas faixas etárias; o dinamismo do cuidado; a alta taxa de letalidade; o uso ininterrupto de EPI's causando lesões; e a limitação da comunicação com os pacientes. Souza et. al (2021), ressaltam que um dos sentimentos que abalou a população e mais intensamente aos profissionais na linha de frente ao combate à COVID-19 foi o medo da infecção pelo SARS-CoV-2, instigado pelo temor à doença e suas complicações e de ser veículo de contaminação para seus familiares.

O estresse e o desgaste profissional dos enfermeiros estão diretamente conectados com: o cuidado ao paciente hemodinamicamente instável; o contato rotineiro com a morte e com o sofrimento dos familiares e pacientes; o quantitativo de arsenal tecnológico, o ambiente e a alta sobrecarga de trabalho (Fernandes Souza et al., 2018). E, todos esses fatores são acentuados diante de uma situação pandêmica.

Ramos-Toescher et al. (2020), fala que essa pressão externa e interna faz surgir o medo e a incerteza os quais influenciam de forma negativa no comportamento e bem-estar geral desses profissionais e, consequentemente, interfere na sustentação da qualidade dos cuidados em saúde destinados aos pacientes.

Com uma maior exposição ao risco de infecção de SARS-CoV-2, há uma redução da capacidade física e psicológica dos profissionais da saúde. tal fato acentua-se no âmbito de UTI, estando associado à alta pressão psicológica, causadas pelas intercorrências que ocorrem no dia-a-dia de assistência ao paciente crítico e aspectos da rotina assistencial inerentes a este setor que é mais agitado. Sendo assim, as instituições de saúde devem assegurar a atuação profissional, minimizando os riscos, por meio de medidas protetivas, somadas à vacinação de todos os profissionais de saúde. Desta forma, ter uma atenção às necessidades dos que atuam na UTI é fundamental para garantir os cuidados aos pacientes críticos com COVID-19, salientando a necessidade de cuidar do cuidador (Fernandez-Castillo et al.; Maciel \& Quaresma, 2021). 
O A01 também deixa explícito que, as atribuições exclusivas da enfermagem como o cuidado contínuo e complexo ao paciente, deram a ela um destaque maior na pandemia do coronavírus. Foi percebido que os riscos psicológicos que uma pandemia acarreta não podem ser evitados em sua totalidade, no entanto, podem ser atenuados a partir do exercício de resiliência, com o objetivo de auxiliar no enfrentamento dos desafios da assistência prestada pela equipe de Enfermagem. Esse é o pontochave da discussão levantada no A02 e, de acordo com Brolese et al. (2017), no ambiente de trabalho, a resiliência caracterizase pelo crescimento pessoal e profissional, a fim de desenvolver habilidades impostas pela profissão.

Ou seja, no contexto pandêmico, o trabalhador precisa reconhecer suas limitações, elencar competências com vistas a melhorar sua atuação e saber enfrentar as adversidades encontradas no cotidiano a fim de manter sua saúde mental. Saurusaitis et al. (2020), considera o apoio a esses profissionais em suas necessidades, uma das ações com maior impacto para a qualidade da assistência em terapia intensiva.

Marins et al. (2020), ressalta que relacionado ao estresse, surgem fatores que auxiliam no desgaste físico e mental, como por exemplo, condições de trabalho precárias, altas jornadas e sobrecarga de trabalho, exposição a fatores de riscos, desmotivação profissional, baixa remuneração e dupla jornada de serviços, o que resulta em reflexos negativos na qualidade de vida desse profissional e também já foram citados pelos artigos utilizados na pesquisa.

Góes et al. (2020) afirma que, tanto pela inadequação estrutural e más condições de trabalho quanto pelas incertezas que permeiam os mecanismos de transmissibilidade e patogenicidade da COVID-19, levando em consideração que é uma doença recente e de larga escala, origina-se no prestador de cuidados, o medo do contágio, próprio e de seus familiares.

Além do pouco tempo de preparação para capacitação, poucos recursos de cuidados intensivos (material e humano), tempo hábil para treinamento de profissionais atuantes em outros setores e áreas, citados no A02 como desafios bem característicos vividos na pandemia, vêem-se destacadas por Batista e Takashi (2020), situações como: a alta quantidade de perdas de vidas, lidar com más notícias, gerenciar grandes escalas, estabelecer novas maneiras de comunicação através da tecnologia, medo da morte de parentes e a predisposição à depressão e ao estresse agudo prolongado, as quais já faziam parte do cotidiano da equipe de enfermagem da UTI, entretanto, se intensificaram com o surgimento do novo coronavírus.

A comunicação de más notícias relaciona-se às situações que podem modificar negativamente, parcialmente ou radicalmente o futuro da vida das pessoas envolvidas - paciente, família, comunidade. Dessa maneira ressalta-se a importância da qualidade do processo de comunicação de más notícias por todos os profissionais que compõem a equipe multidisciplinar de saúde, em todos os níveis de atenção e de complexidade do atendimento à população (Fontes et al., 2017). Com a pandemia da COVID-19, a comunicação ficou prejudicada devido ao distanciamento social e ao uso de EPIs, tornando o momento da notícia mais conturbado.

Assim como no A01 e A02, no A03 ficaram evidentes dificuldades semelhantes enfrentadas pela equipe de Enfermagem na UTI na assistência, como a minimização do contato com o paciente e a falta de proximidade que prejudicam o desempenho ideal de cuidado. $\mathrm{O}$ aumento na carga de trabalho e a falta de especialização dos Enfermeiros em UTI foram pontos bem citados pelos enfermeiros em questão. Sobretudo, o medo foi a emoção mais relatada por esses profissionais, o qual regia todas as situações do ambiente de trabalho e tomadas de decisão necessárias.

De acordo com Teixeira et al. (2020), o medo citado no A03 é intensificado pela má gestão e falta de EPI's, pois, dessa forma, há uma exposição maior ao vírus. O medo não só do adoecimento, como também de contaminar familiares e amigos está diretamente ligado ao desgaste e ao estresse mental já citados anteriormente. Brasileiro e Brasileiro (2017) abordam que tanto na área da saúde quanto nas ciências humanas, não há preparo suficiente para se lidar com os medos que surgem durante o processo de morte com o surgimento da pandemia essas situações ficaram ainda mais intensas. 
Chen et al. (2020), destaca que profissionais da enfermagem vêm se preocupando muito com questões como escassez de equipamentos de proteção individual, passandoa considerar as intervenções psicológicas como secundárias ou sem prioridade. Assim, para psicólogos que atuam em hospitais e outros serviços de saúde, sugere-se a realização de visitas à área de descanso para escutar os desafios vivenciados por eles.

Em relação a sobrecarga de trabalho, Da costa et al. (2018) traz que essa, combinada à longa jornada de trabalho, pode provocar fragilidades na habilidade funcional e moral dos enfermeiros podendo torná-los insatisfeitos, sem vontade de permanecer no trabalho, aumentar os casos de depressão, sofrimento e sinais físicos. Além disso, com a sobrecarga de trabalho e a pouca valorização do seu serviço, o enfermeiro apresenta-se não só insatisfeito com a profissão mas também com o ambiente de trabalho. Borges et al. (2017), afirmou que o aumento da carga de trabalho da equipe de enfermagem - comumente associado ao déficit na equipe - apresenta resultados alarmantes para o atendimento ao paciente, tais como: o aumento do tempo de hospitalização; a elevação das taxas de infecção relacionada a cuidados, lesões por pressão, quedas e erros na administração de medicamentos; e ainda maior propensão à morte.

Os temas "ineficiência da organização no apoio aos enfermeiros", "esgotamento físico", "vivendo com incertezas" e "carga psicológica da doença", foram abordados nas entrevistas com enfermeiros no decorrer do A04. A falta de apoio financeiro e escassez de mão de obra aliada a turnos pesados sem licença, reforçam a desvalorização dos profissionais da Enfermagem.

Segundo Laitano et al. (2019), essa desvalorização e a precarização das condições de trabalho são marcas na história da Enfermagem e estão ligadas diretamente com a sua origem que é advinda dos afazeres domésticos e majoritariamente exercida por mulheres. O engajamento político da classe e a divulgação da importância da profissão na mídia a partir do destaque da atuação da Enfermagem na linha de frente durante a pandemia, foram bons caminhos para iniciar a luta contra essa desvalorização.

A quantidade insuficiente de EPI's e a discriminação na distribuição destes também foram destacadas por esses profissionais no A04. Essa discriminação se caracterizou por os EPI's com melhores qualidades e em maiores quantidades serem distribuídos para os médicos, fomentando uma situação de desigualdade no setor da UTI. Lage e Alves (2017) afirmam que os Enfermeiros consideram seu trabalho e sua presença apagados quando comparados ao trabalho do médico. A visão que a sociedade tem da enfermagem ser inferior à medicina é explicada pela caracterização do médico como 'mais intelectual', e que simbolicamente, detém o 'poder da cura'.

Na realidade dos Enfermeiros que responderam o questionário do A05, pode-se salientar alguns tópicos importantes a partir dos relatos, como: a insuficiência no fornecimento de alimentos comparado ao desgaste físico do trabalho; não conseguirem atender às necessidades psicoemocionais dos familiares; falta de preocupação dos gerentes com a necessidade das equipes de atendimento; e deram ênfase à grande exaustão emocional ao fim da jornada de trabalho.

Enfermeiros com especialização declararam ter menos medo de cometer erros e tinham um maior conhecimento comparados a enfermeiros generalistas. Almeida et al. (2019) cita que o Enfermeiro recém-formado é novato no campo de atuação, visto que não tem experiência e/ou domínio do cuidado especializado, mesmo que tenha experiência em outra área. As pesquisas que descrevem as características do Enfermeiro de UTI no que se refere ao cuidado intensivo, apontam limitações dos Enfermeiros generalistas na tomada de decisão clínica e dificuldades para cuidar com segurança. E isso se deve pela distinção da teoria com a realidade da assistência.

Desse modo, é visível a escassez de Enfermeiros com especialização em assistência em Unidade de Terapia Intensiva e isso ficou mais evidente com a ascensão da pandemia de COVID-19. A02, A03 e A05 reafirmam essa carência a partir da exposição dessa difícil realidade através de seus relatos. 
Apesar das inúmeras dificuldades enfrentadas pela equipe de enfermagem expostas acima, além daquelas que não foram contempladas nos artigos encontrados, A03 e A05 trazem a melhoria do trabalho em equipe, nas relações de trabalho e o trabalho interdisciplinar que antes não era costume. Esse ponto positivo se deu devido à necessidade de união para enfrentar essa situação pandêmica. O A04 ainda fala que a pandemia serviu para explorar as habilidades do Enfermeiro em saber separar as emoções pessoais das dos pacientes e ser líder, tendo espírito de equipe.

A atuação do enfermeiro enquanto líder de equipe na área hospitalar tem ocorrido sem suporte das instituições, pautados apenas na experiência prática dos profissionais, com pouco resgate de embasamento teórico e com poucos instrumentos que norteiam os principais tipos de liderança, configurando assim, um empecilho no aperfeiçoamento da liderança (Scofano, Valente \& Lanzillotti, 2018).

A partir da análise dos artigos selecionados, pôde-se perceber que atuar na linha de frente requer muitas habilidades aliadas à resiliência para conseguir enfrentar os desafios. É evidente a importância de valorizar a enfermagem e dá-lhes suporte psicológico, financeiro e estrutural para que a assistência seja efetiva e completa. O maior grupo da área de saúde precisa de destaque e auxílio para superar obstáculos e continuar atuando de maneira singular tanto na pandemia de COVID-19 quanto no cuidado fora do contexto pandêmico, em especial na UTI, prestando serviço aos que mais precisam, que são os pacientes com alto grau de complexidade.

\section{Conclusão}

A pandemia do coronavírus vem marcando a história da humanidade e impactando diretamente na vida das pessoas, além de acarretar uma considerável sobrecarga aos profissionais de saúde. A equipe de Enfermagem destaca-se nesse combate ao COVID-19 por estar na linha de frente, sendo atingida de diferentes maneiras nos âmbitos físico, psicológico e social. Essa situação se torna mais complexa na UTI, visto que já é um ambiente com grande carga psicológica.

Dessa forma, é de extrema necessidade evidenciar e debater os principais pontos negativos que interferem no desempenho profissional desses trabalhadores visto que também afeta diretamente a recuperação dos pacientes críticos. Os desafios diários são inúmeros, como: escassez de mão de obra qualificada e especializada, carência de EPIs, cargas de trabalho abusivas, estresse, medo de contaminação, falha na comunicação com o paciente, exaustão física e emocional, entre outros. Contudo, saber lidar com eles de maneira a afetar o mínimo possível o desempenho e vivência desse profissional deve ser prioridade nesse momento. Cuidar e dar suporte a estes profissionais é essencial para alcançar resultados positivos nessa situação atual.

Entretanto, é perceptível que o pequeno número de artigos que abordam essa temática ainda não é o suficiente para explanar todas as dificuldades que a equipe de enfermagem enfrenta no cuidado ao paciente com COVID-19 na UTI, devido a singularidade das circunstâncias de cada local do mundo, tornando assim, imprescindível um aprofundamento maior e mais pesquisas que tenham foco de melhorar a qualidade de trabalho desses profissionais.

Acolher e valorizar a equipe de Enfermagem, dando-lhes condições favoráveis de trabalho, sejam elas materiais e estruturais, amparo psicológico e jornadas de trabalho adequadas, a partir da contratação de pessoal especializado, configuram ações positivas para favorecer um bom desempenho desses que estão na linha de frente de uma das maiores pandemias da história.

A partir disso, é imprescindível que haja mais pesquisas sobre esse tema, devido à importância atual e por ainda ser escassa essa abordagem específica à assistência a pacientes com COVID-19 na UTI. Uma maior quantidade de estudos nessa linha trará frutos inestimáveis para o futuro da saúde mundial. 


\section{Referências}

Almeida, R. O., Oliveira, F. T., Ferreira, M. A. \& Silva, R. C. (2019). Newly undergraduate nurses and intensive care in units of non-critical patients. Revista Brasileira de Enfermagem. 72(1), 243-51, fev. FapUNIFESP (SciELO). http://dx.doi.org/10.1590/0034-7167-2017-0713.

Araujo, A. S. \& Comassetto, I. (2021). The nursing protagonism in the organization of health services during the COVID-19 pandemic. Research, Society and Development. 10(1), e48110112014. https://rsdjournal.org/index.php/rsd/article/view/12014.

Arnetzs, J. E., Goetz, C. M., Arnetz, B. B. \& Arble, E. (2020). Nurse Reports of Stressful Situations during the COVID-19 Pandemic: qualitative analysis of survey responses. International Journal Of Environmental Research And Public Health. 17(21), 8126. MDPI AG. http://dx.doi.org/10.3390/ijerph17218126.

Batista, L. S. \& Takashi, M. H. (2020). Os principais fatores causadores de Estresse em profissionais de enfermagem que atuam em Unidade de Terapia Intensiva. Revista de Divulgação Científica Sena Aires, 156-162, abr. Revista de Divulgação Científica Sena Aires. http://dx.doi.org/10.36239/revisa.v9.n1.p156a162.

Bitencourt, J. V. O. V., Meschial, W. C., Frizon, G., Biffi, P., Souza, J. B. \& Maestri, E. (2020). Nurse's protagonism in structuring and managing a specific unit for covid-19. Texto \& Contexto - Enfermagem, 29, 1-11. FapUNIFESP (SciELO). http://dx.doi.org/10.1590/1980-265x-tce-2020-0213.

Borges, E. M. N., Queirós, C. M. L., Vieira, M. R. F. S. P. \& Teixeira, A. A. R. (2021). Perceptions and experiences of nurses about their performance in the COVID-19 pandemic. Rev Rene, 22, 1-9, jan. Rev Rene - Revista da Rede de Enfermagem de Nordeste. http://dx.doi.org/10.15253/2175-6783.20212260790.

Borges, F., Borher, C. D., Bugs, T. V., Nicola, A. L, Tonini, N. S. \& Oliveira, J. L. C. (2017). Dimensionamento de pessoal de enfermagem na UTI-Adulto de hospital universitário público. Cogitare Enfermagem, 22 (2). https://www.redalyc.org/jatsRepo/4836/483654815018/483654815018.pdf.

Brasileiro, M. S. E. \& Brasileiro, J. E. (2017). O medo da morte enquanto mal: uma reflexão para a prática da enfermagem. Revista de Ciências Médicas, 26 (2), 77, nov. https://doi.org/10.24220/2318-0897v26n2a3582.

Brolese, D. F., Lessa, G., Santos, J. L. G., Mendes, J. S., Cunha, K. S. \& Rodrigues, J. (2017). Resilience of the health team in caring for people with mental disorders in a psychiatric hospital. Revista da Escola de Enfermagem da Usp, 51, 1-8, ago. Fap UNIFESP (SciELO). http://dx.doi.org/10.1590/s1980$220 \times 2016026003230$.

Cadernos de Fe e Cultura, Oculum Ensaios, Reflexão, Revista de Ciencias Medicas e Revista de Educação da PUC-Campinas. http://dx.doi.org/10.24220/2318$0897 \mathrm{v} 26 \mathrm{n} 2 \mathrm{a} 3582$

Campos, F. C. C. \& Canabrava, C. M.(2020). O Brasil na UTI: atenção hospitalar em tempos de pandemia. Saúde em Debate, 1-22, out. FapUNIFESP (SciELO). http://dx.doi.org/10.1590/scielopreprints. 1368 .

Chen, Q., Liang, M., Li, Y., Guo, J., Fei, D., Wang, L., He, L., Sheng, C., Cai, Y. \& Li, X. (2020). Mental health care for medical staff in China during the COVID-19 outbreak. The Lancet Psychiatry, 7 (4), 15-16, abr. Elsevier BV. http://dx.doi.org/10.1016/s2215-0366(20)30078-x.

Cunha, I. C. K. O., Erdmann, A. L., Balsanelli, A. P., Cunha, C. L. F., Lopes, D. N., Ximenes, R. G. N., Santos, J.L. G. \& Lourenção, L. G. (2020). Ações e estratégias de escolas e departamentos de enfermagem de universidades federais frente à COVID-19. Enferm. foco (Brasília), 11 (1) 4857. http://revista.cofen.gov.br/index.php/enfermagem/article/view/4115/802.

Da Costa, C. S., Normann, K. A. S., Tanaka, A. K. S. R. \& Cicolella, D. A. (2018). A influência da sobrecarga de trabalho do enfermeiro na qualidade da assistência. revista uningá, 55 (4), 110-120, dez. http://revista.uninga.br/index.php/uninga/article/view/2403.

De Sousa, A. R., Santos, G. L. A., SIlva, R. S. \& Carvalho, E. S. S. (2020). Reflexões sobre o Processo de Enfermagem no trabalho de enfermeiras frente à pandemia da COVID-19. Enfermagem em Foco, 1-6, jun. http://revista.cofen.gov.br/index.php/enfermagem/article/view/3501/804

De Sousa, L. M. M., Marques-Vieira, C. M. A., Severino, S. S. P. \& Antunes, A. V. (2017). A metodologia de revisão integrativa da literatura em enfermagem. Revista Investigação em Enfermagem, 17-26, nov. http://www.sinaisvitais.pt/images/stories/Rie/RIE21.pdf\#page=17.

Fernandez, R., Lord, H., Halcomb, E., Mohham, L., Middleton, R., Alanzech, I. \& Ellwood, L. (2020). Implications for COVID-19: a systematic review of nurses :: experiences of working in acute care hospital settings during a respiratory pandemic. International Journal Of Nursing Studies, 111, 103637, nov. Elsevier BV. http://dx.doi.org/10.1016/j.ijnurstu.2020.103637.

Fernandez-Castillo, R-J., Gonzalez-Caro, M-D., Fernandez-Garcia, E., Porcel-Galvez, A-M. \& Garnacho-Monteiro, J (2021). Intensive care nurses' experiences during the COVID -19 pandemic: a qualitative study. Nursing In Critical Care, 1-10, jan. Wiley. http://dx.doi.org/10.1111/nicc.12589.

Fontes, C. M. B., Menezes, D. V., Borgato, M. H., \& Luiz, M. R. (2017). Communicating bad news: an integrative review of the nursing literature. Revista Brasileira de Enfermagem, 70 (5), 1089-1095, out. FapUNIFESP (SciELO). <http://dx.doi.org/10.1590/0034-7167-2016-0143.

Gerolin, F. S., Pires, A. M., Nascimento, C., Schimitt, C., Bucione, F. T. S., Rocha, J. S. A., Berlofi, L. M. \& Ferrari, L. C. S. (2020). AÇÕES DE LIDERANÇAS DA ENFERMAGEM NA ORGANIZAÇÃO DO ATENDIMENTO HOSPITALAR A PACIENTES COM COVID-19. Enfermagem em Foco, 207-211, abr. http://revista.cofen.gov.br/index.php/enfermagem/article/view/3665.

Góes, F. G. B., Silva, A. C. S. S., Santos, A. S. T., Pereira-Ávila, F. M. V., Silva, L. J., SIlva, L. F. \& Goulart, M. C. L. (2020). Challenges faced by pediatric nursing workers in the face of the COVID-19 pandemic. Revista Latino-Americana de Enfermagem, 28, 1-9. FapUNIFESP (SciELO). http://dx.doi.org/10.1590/1518-8345.4550.3367

Gonzalez-Gil, M. T., Gonzalez-Blazquez, C., Parro-Moreno, Aa. I., Pedraz-Marcos, A., Palmas-Santos, A., Otero-Garcia, L., Navarta-Sanchez, M. V., AlcoleaCosin,, M. T., Arguello-Lopez, M. T. \& Canalejas-Perez, C. (2021). Nurses' perceptions and demands regarding COVID-19 care delivery in critical care units and hospital emergency services. Intensive And Critical Care Nursing, 62, 102966, fev. Elsevier BV. http://dx.doi.org/10.1016/j.iccn.2020.102966 
Lage, C. E. B. \& Alves, M. S. (2017). Debatendo a valorização da enfermagem: a voz dos enfermeiros da atenção primária à saúde. Rev. enferm. UFPE on line, 1381-1387. https://pesquisa.bvsalud.org/portal/resource/pt/biblio-1032419.

Laitano, A. C., Silva, G. T. R., Almeida, D. B., Santos, V. P. F. A., Brandão, M. P., Carvalho, A. G., Peres, M. A. A. \& Santana, N. (2019). Precarização do trabalho da enfermeira: militância profissional sob a ótica da imprensa. Acta Paulista de Enfermagem, 32 (3), 305-311, jun. FapUNIFESP (SciELO). http://dx.doi.org/10.1590/1982-0194201900042.

Lee, N. \& Lee, H-J. (2020). South Korean Nurses' Experiences with Patient Care at a COVID-19-Designated Hospital: growth after the frontline battle against an infectious disease pandemic. International Journal Of Environmental Research And Public Health, 17 (23), 9015, dez. http://dx.doi.org/10.3390/ijerph17239015.

Lima, L. S., Bessa, M. M., Silva, S. W. S., Moura, K. M., Souza, J. O. \& Freitas, R. J. M. (2021). processo de enfermagem para pacientes com manifestações respiratórias da covid-19. Revista de Enfermagem Ufpe On Line, 15 (1), 1-9, jan. http://dx.doi.org/10.5205/1981-8963.2021.245345.

Maciel, E. S. \& Quaresma, F. R. P. (2021). Cadernos Educativos: vacinação contra a COVID 19. https://scholar.google.com.br/scholar?hl=ptBR\&as_sdt=0\%2C5\&q=Cadernos+Educativos\%3A+vacina\%C3\%A7\%C3\%A3o+contra+a+COVID+19.\&btnG=.

Marins, T. V. O., Crispim, C. G., Evangelista, D. S., Neves, K. C., Fassarella, B. P. A., Ribeiro, W. A., Silva, A. A. (2020). Enfermeiro na linha de frente ao COVID-19: a experiência da realidade vivenciada. Research, Society And Development, 9 (8), 1-22, jul. http://dx.doi.org/10.33448/rsd-v9i8.6471.

Marmelstein, G. \& Morozowski, A. C. (2020). Que Vidas Salvar? Escassez de Leitos de UTI, Critérios Objetivos de Triagem e a Pandemia da COVID-19. Revista Publicum, 6 (1), 94-117. https://www.e-publicacoes.uerj.br/index.php/publicum/article/view/57573/37411.

Melo, G. A. A., Silva, R. A., Aguiar, L. L., Medina, L. A. C., Oliveira, C. V. F. \& Caetano, D. G. M. J. A.. (2019). relationship between professional profile of intensive care nurses and missed care in hemodialysis therapy. Reme Revista Mineira de Enfermagem, 23, 1-9, ago. GN1 Genesis Network. http://dx.doi.org/10.5935/1415-2762.20190113.

Mendes, K. S., Silveira, R. C. C. P., Galvão, C.M. (2019). USE OF THE BIBLIOGRAPHIC REFERENCE MANAGER IN THE SELECTION OF PRIMARY STUDIES IN INTEGRATIVE REVIEWS. Texto \& Contexto - Enfermagem, 28, 1-13. FapUNIFESP (SciELO). http://dx.doi.org/10.1590/

Moradi, Y., Bagahei, R., Hosseingholipour, K. \& Mollazadeh, F. (2021). Challenges experienced by ICU nurses throughout the provision of care for COVID19 patients: a qualitative study. Journal Of Nursing Management, 29 (5), 1159-1168, fev. http://dx.doi.org/10.1111/jonm.13254

Nedel, W. L., Silveira, F. (2016). Different research designs and their characteristics in intensive care. Revista Brasileira de Terapia Intensiva, 28 (3), 1-5. GN1 Genesis Network. http://dx.doi.org/10.5935/0103-507x.20160050.

Ouchi, J. D., Luo, A. P. R., Alves, B. O., Andrade, R. V. \& Fogaça, M. B. (2018). O PAPEL DO ENFERMEIRO NA UNIDADE DE TERAPIA INTENSIVA DIANTE DE NOVAS TECNOLOGIAS EM SAÚDE. Revista Saúde em Foco, 10, 412-428. http://portal.unisepe.com.br/unifia/wpcontent/uploads/sites/10001/2018/07/054_O_PAPEL_DO_ENFERMEIRO_NA_UNIDADE_DE_TERAPIA_INTENSIVA.pdf .

Paixão, G. L. S., Freitas, M. I., Cardoso, L. C. C., Carvalho, A. R., Fonseca, G. G., Andrade, A. F. S. M., Passos, T. S. \& Torres, R. C. (2021). estratégias e desafios do cuidado de enfermagem diante da pandemia da covid-19/ strategies and challenges of nursing care in the face of covid-19 pandemic. Brazilian Journal Of Development, 7 (2), 19125-19139, jan . http://dx.doi.org/10.34117/bjdv.

Ramalho Neto, J. M.., Viana, R. A. P. P., Franco, A. S., Prado, P. R.G., F. A. F. \& Nóbrega, M. M. L. (2020). NURSING DIAGNOSIS/OUTCOMES AND INTERVENTIONS FOR CRITICALLY ILL PATIENTS AFFECTED BY COVID-19 AND SEPSIS. Texto \& Contexto - Enfermagem, 29, 1-17. FapUNIFESP (SciELO). http://dx.doi.org/10.1590/1980-265x-tce-2020-0160.

Ramos-Toescher, A. M., Tomaschewisk-Barlem, J. G., Barlem, E. L. D., Castanheira, J. S., Toescher, R. L. (2020). Saúde mental de profissionais de enfermagem durante a pandemia de COVID-19: recursos de apoio. Escola Anna Nery, 24, 1-7, ago. FapUNIFESP (SciELO). http://dx.doi.org/10.1590/21779465-ean-2020-0276

Rocha, N. H. G. \& Lemos, R. C. A. (2017). atitudes da equipe e qualidade da assistência de enfermagem em um pronto socorro adulto. Revista de Enfermagem $\begin{array}{lllll}e & \text { Atenço } & \text { Saúde, } & \text { Uberlândia, } & 6(20,\end{array}$ https://web.archive.org/web/20180421062309id_/http://seer.uftm.edu.br/revistaeletronica/index.php/enfer/article/viewFile/1842/pdf.

Saurusaitis, A. D., Vieira, R. F. C., Peregrino, A. A. de F., Santo, F. H. do E., Pereira, V. R. F., Silva, R. C. L. da. (2020). Challenges of nursing management in oncology intensive care during the COVID-19 pandemic. Research, Society and Development, 9 (7), 1-18, jun. https://rsdjournal.org/index.php/rsd/article/view/4904.

Scofano, B. S. \&Valente, G. S. C., Lanzillotti, R. S. (2019). Atuação do enfermeiro enquanto líder de equipe na área hospitalar: uma revisão integrativa. Revista Nursing, 22 (253), 2943-2948. http://www.revistanursing.com.br/revistas/253/pg51.pdf

Souza, R. F., Rosa, R. S., Picanço, C. M., Souza, E. V., Cruz, D. P., Guimarães, F. E. O. \& Boery, R. N. S. O. (2018). Repercussões dos fatores associados à qualidade de vida em enfermeiras de unidades de terapia intensiva. Revista de Salud Pública, 20 (4), 453-459, jul. Universidad Nacional de Colombia. http://dx.doi.org/10.15446/rsap.v20n4.65342.

Souza, W. S. de ., Comassetto, I.; Junqueira, T. L. S. ., Souza, E. M. S. de, Oliveira, A. dos S. \&Leão, A. L. (2021). Experience of the Multiprofessional Health Team in coping with COVID-19 in Hospitalization Services. Research, Society and Development, 10 (4), 1-15, abril. https://rsdjournal.org/index.php/rsd/article/view/14048 . 
Research, Society and Development, v. 10, n. 12, e417101220296, 2021

(CC BY 4.0) | ISSN 2525-3409 | DOI: http://dx.doi.org/10.33448/rsd-v10i12.20296

Teixeira, C. F. S., Soares, C. M., Souza, E. A., Lisboa E. E., Pinto, I. C. M., Andrade, L. R.. \& Espiridão, M. A. (2020). A saúde dos profissionais de saúde no enfrentamento da pandemia de Covid-19. Ciência \& Saúde Coletiva, 25(9), 3465-3474, set. FapUNIFESP (SciELO). http://dx.doi.org/10.1590/141381232020259.19562020

Thusini, S. (2020). Critical care nursing during the COVID-19 pandemic: a story of resilience. British Journal Of Nursing, 29 (21), 1232-1236, nov. Mark Allen Group. http://dx.doi.org/10.12968/bjon.2020.29.21.1232. 